Results Compared to the urban group, rural dwellers had a greatly increased likelihood of possible/definite angina (multi-adjusted OR 2.82 (1.68 to 4.73)). Urban and migrant groups had higher levels of risk factors (eg, smoking - 20.1\% urban, 5.5\% rural). No diabetes was seen in the rural dwellers who complained of possible/definite angina. Rural dwellers had a higher prevalence of mood disorder and the presence of a mood disorder was associated with possible/definite angina in all three groups, but not consistently with nonexertional chest pain.

Conclusion Rural groups had a higher prevalence of angina as measured by Rose questionnaire than migrants and urban dwellers, and a higher prevalence of mood disorder. The presence of a mood disorder was associated with angina. The Rose angina questionnaire may not be of relevance to rural populations in developing countries with a low pre-test probability of coronary disease and poor mental health.

\section{P2-342 GEOGRAPHICAL PATTERN AND EPIDEMIOLOGY OF STOMACH CANCER MORTALITY IN IRAN}

\section{doi:10.1136/jech.2011.142976k.74}

\author{
${ }^{1,2} \mathrm{~K}$ Zendehdel, ${ }^{*}{ }^{1} \mathrm{M}$ Marzban, ${ }^{1} \mathrm{~A}$ Nahvijou, ${ }^{1} \mathrm{~N}$ Jafari. ${ }^{1}$ Cancer Research Center, \\ Cancer Institute of Iran, Tehran, Iran; ${ }^{2}$ Department of Medical Epidemiology and \\ Biostatistcs, Karolinska Institutet, Stockholm, Sweden; ${ }^{3}$ Health Network Development \\ Center, Ministry of Health and Medical Education, Tehran, Iran
}

Background Stomach cancer is the second commonest cause of mortality from cancer worldwide; in Iran it is the commonest. Geographical variation in the incidence of stomach cancer is reported. Data describing the geographical distribution of disease in Iran are lacking. The aim of this study was to examine geographical variation in stomach cancer mortality in Iran.

Methods We used the Iranian National Causes of Death Registry and estimated age-standardised mortality rates (ASMR) of stomach cancer in 29 provinces, using the age distribution of the world standard population, stratified by sex and residential area (rural/urban).

Results ASMRs of stomach cancer were 15 per 100000 in men and 8 per 100000 in women. The highest and lowest mortality rates were observed in Kordestan (ASMR=29.1 per 100000 ) and Hormozgan $(A S M R=5.0$ per 100000$)$ provinces in the north-western and southern Iran, respectively. The mortality rates were approximately twice as high in men and rural residents as women and urban residents respectively.

Conclusions The incidence of stomach cancer in Iran is a high with evidence of regional variation. The substantial variation in stomach cancer mortality rates between northern and southern Iran warrants further investigation. The results of this study can be used for resource allocation and to inform the designing of appropriate gastric cancer control programs in Iran.

\section{P2-343 EFFECTS ANALYSIS OF THE PREVENTIVE MEASURES ON NEURAL TUBE DEFECTS IN CHINA}

doi:10.1136/jech.2011.142976k.75

Z Zhao, ${ }^{*}$ G Rui, Z Wang, L Gao, 0 Lu, X Sun. Department of Epidemiology, School of Public Health, Shandong University, Jinan, Shandong, China

Introduction China is among the high incidence countries for neural tube defects (NTDs) and great efforts have been made towards their prevention. This study is to understand the effects and influencing factors on prevention measures.
Methods A matched case-control study and prevalence studies were employed. 459 women who gestated babies/foetuses with NTDs and their controls were selected and investigated in 24 districts of two provinces in China. Logistic regression models and interaction analysis was used for data analysis.

Results Folic acid supplementation, planned pregnancy, preconception examination and health education were associated with reduced NTDs (ORs 0.52, 0.27, 0.48 and 0.36 respectively). The folic acid supplementation rate was $5.0 \%$ of cases and $17.2 \%$ of controls. Folic acid supplementation showed synergistic interaction effects with the other primary prevention measures and prevention rates were $93 \%, 89 \%$ and $90 \%$ respectively. $85.9 \%$ of the NTDs were diagnosed by ultrasound screening on average at 24.0 weeks gestation. The detection rates by ultrasonography before 16, 16-20, 20-24, 24-28 and after 28 weeks were $14.1 \%$, $49.4 \%, 46.3 \%, 49.2 \%$ and $52.1 \%$ respectively $(\mathrm{p}<0.05)$. The detection rates were $46.4 \%, 52.0 \%$ and $28.1 \%$ in hospitals, maternal and child care service centres and family planning centres respectively $(\mathrm{p}<0.05)$.

Conclusion Folic acid supplementation rate was low in the study subjects. Its use is correlated with planned pregnancy, preconception examination and health education. The efficiency of ultrasonography for NTDs screening could be improved in medical reproduction health institutions.

\section{P2-344 FRAILTY AND MULTIMORBIDITY IN THE ELDERLY: RESULTS FROM THE KORA-AGE AUGSBURG SURVEY}

doi:10.1136/jech.2011.142976k.76

${ }^{1} \mathrm{~A}-\mathrm{K}$ Zimmermann, ${ }^{*} \mathrm{~B}$ Thorand, ${ }^{1,2} \mathrm{C}$ Meisinger, ${ }^{1,2} \mathrm{M}$ Heier, ${ }^{1} \mathrm{~A}$ Peters, ${ }^{1} \mathrm{~A}$ Döring. ${ }^{1}$ Helmholtz Zentrum München, German Research Centre for Environmental Health, Institute of Epidemiology II, Neuherberg, Germany; ${ }^{2}$ Central Hospital of Augsburg, MONICA KORA Myocardial Infarction Registry, Augsburg, Germany

Background This study investigated the prevalence of frailty as well as its association with multimorbidity among older adults living in the South of Germany.

Methods 1079 participants aged 65 years and older were interviewed and took part in a physical examination in the KORAAge survey. Frailty was defined by weight loss, exhaustion, low physical activity, slow gait speed, and reduced grip strength. Participants were classified as non-frail, pre-frail, and frail if they met 0,1 or 2 , and 3 or more criteria, respectively. Multimorbidity was defined in three groups, group 1 with no disease, group 2 with one or two diseases, and group 3 with more than three out of nine disease groups. Multivariable logistic regression analyses adjusted for malnutrition, alcohol intake, education and body mass index were performed.

Results The age standardised prevalence of frailty and pre-frailty combined was $35.6 \%$ (95\% CI 31.1 to 40.0 ) for male and 38.5\% (95\% CI 34.1 to 42.8) for female participants. Prevalence of frailty increased with age from $24.1 \%$ in the $65-69$ year olds to $78.0 \%$ in the over 85 year olds for men (20.7\% to $79.3 \%$ in women). The risk for being frail increased with higher multimorbidity scores (OR 1.9, $95 \%$ CI 1.4 to 2.6 for group 2 and OR $3.3,95 \%$ CI 1.8 to 6.0 for group 3 each compared to group 1) after adjustment for covariables. $27.8 \%$ of pre-frail and frail persons had no disease.

Conclusion These data indicate that frailty is strongly associated with multimorbidity. Examinations of the course of frailty and its determinants are underway. 